\title{
Numerical investigation of a coupled moving boundary model of radial flow in low-permeable stress-sensitive reservoir with threshold pressure gradient*
}

\author{
Wen-Chao Liu(刘文超)，Yue-Wu Liu(刘曰武) ${ }^{\dagger}$, Cong-Cong Niu(牛丛丛), \\ Guo-Feng Han(韩国锋), and Yi-Zhao Wan(万义钊) \\ Institute of Mechanics, Chinese Academy of Sciences, Beijing 100190, China
}

(Received 12 May 2015; revised manuscript received 25 September 2015; published online 10 January 2016)

\begin{abstract}
The threshold pressure gradient and formation stress-sensitive effect as the two prominent physical phenomena in the development of a low-permeable reservoir are both considered here for building a new coupled moving boundary model of radial flow in porous medium. Moreover, the wellbore storage and skin effect are both incorporated into the inner boundary conditions in the model. It is known that the new coupled moving boundary model has strong nonlinearity. A coordinate transformation based fully implicit finite difference method is adopted to obtain its numerical solutions. The involved coordinate transformation can equivalently transform the dynamic flow region for the moving boundary model into a fixed region as a unit circle, which is very convenient for the model computation by the finite difference method on fixed spatial grids. By comparing the numerical solution obtained from other different numerical method in the existing literature, its validity can be verified. Eventually, the effects of permeability modulus, threshold pressure gradient, wellbore storage coefficient, and skin factor on the transient wellbore pressure, the derivative, and the formation pressure distribution are analyzed respectively.
\end{abstract}

Keywords: threshold pressure gradient, stress-sensitive effect, wellbore storage, skin effect

PACS: 47.11.Bc, 47.56.+r, 02.70.Bf

DOI: $10.1088 / 1674-1056 / 25 / 2 / 024701$

\section{Introduction}

In modern times, these unconventional tight oil and gas reservoirs such as oil \& gas shale reservoirs and lowpermeable reservoirs have become new energy targets in petroleum engineering; and they play more and more important roles in the world's energy consumption. Correspondingly, the studies of the kinematic principles of fluid flow in unconventional reservoirs and their applications in engineering problems have become a hot topic at present.

\subsection{Moving boundary problem considering threshold pressure gradient}

Abundant experimental and theoretical analyses ${ }^{[1-13]}$ have demonstrated that the fluid flow in low-permeable reservoirs do not obey the conventional Darcy's law; there exists a threshold pressure gradient (TPG). That is, the fluid flow happens only if the formation pressure gradient exceeds TPG. In particular, $\mathrm{Cai}^{[11]}$ presented the analytical expressions for the flow rate and the velocity of non-Newtonian fluid flow in lowpermeable porous medium, and also obtained the scaling relation between TPG and permeability through fractal approach.

Unlike the modeling of Darcy's flow, the flow in porous medium with TPG is a nonlinear moving boundary problem. Figure 1 shows the computed dimensionless formation pres- sure distribution curves corresponding to Darcy's flow and the flow in porous medium with TPG, respectively. Compared with the result of Darcy's flow, the dimensionless formation pressure distribution curve of modified Darcy's flow is much steeper, and the dimensionless formation pressure becomes zero value at the position of moving boundary. At the moving boundary, the formation pressure gradient is just equal to TPG. Inside the moving boundary, the dimensionless formation pressure gradient is larger than TPG, and then the fluid flow can be driven under a net pressure gradient, i.e., the formation pressure gradient minus TPG. However, outside the moving boundary, no fluid flow happens (the formation pressure keeps initial pressure; and the formation pressure gradient is equal to zero, which is a "jump" of pressure gradient at the moving boundary position). In a word, the formation pressure curve corresponding to modified Darcy's flow shows a property of compact support. ${ }^{[10]}$

In another way, if an angle $\alpha$ between formation pressure curve and distance is set to be on the moving boundary, TPG will be equal to the value of tangent $\alpha$ from the definition of TPG. When TPG tends to zero, the tangent of $\alpha$ will become zero gradually. Then the type of formation distribution curve will tend to a limit case i.e., Darcy's flow.

*Project supported by the National Natural Science Foundation of China (Grant No. 51404232), the China Postdoctoral Science Foundation (Grant No. 2014M561074), and the National Science and Technology Major Project, China (Grant No. 2011ZX05038003).

†Corresponding author. E-mail: liuyuewulxs@126.com

(C) 2016 Chinese Physical Society and IOP Publishing Ltd

http://iopscience.iop.org/cpb http://cpb.iphy.ac.cn 


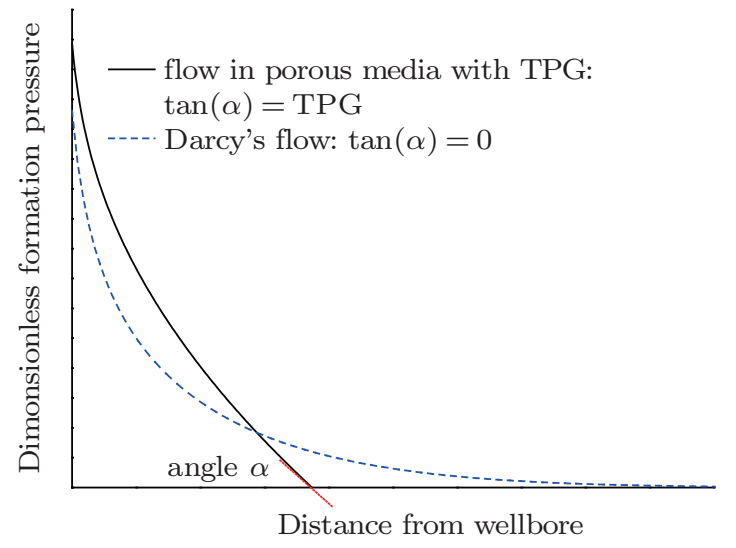

Fig. 1. (color online) Comparison between computed dimensionless formation pressure distribution curves with and without including TPG.

Many relevant studies have proved that it is very significant to take into account the TPG in the modeling of fluid flow in porous medium in low-permeable reservoir, whether from reservoir engineering applications or theoretical modeling analysis. For example, the gas flow in water bearing tight gas reservoirs in consideration of TPG is investigated by Zhu et $a l .{ }^{[14]}$ and through the analytical investigation, their presented mathematical model shows that due to the existence of TPG the reservoir energy is largely consumed near the wellbore. The research ${ }^{[15]}$ by Zhu et al. shows that the considering of TPG in low-permeable reservoir simulation can improve the history matching precision in the relevant applications in Units X10 and X11 of Daqing Oilfield. In Yin and Pu's study, ${ }^{[16]}$ it is demonstrated through a pilot test in Chao 45 Block of Daqing Oilfield that the improved history matching degree can be reached under the condition of considering TPG in the simulation of surfactant flooding in low-permeable reservoir. Yao et al. ${ }^{[10]}$ and Liu et al. ${ }^{[17]}$ investigated a basic one-dimensional moving boundary problem of fluid flow in porous medium with TPG by an analytical solution method and a subsequent strictly verified numerical method. And it is concluded that it is very necessary to take into account the effect of TPG for the fluid flow in porous medium with TPG, for mathematical modeling in engineering applications.

\subsection{Stress-sensitive effect}

In the actual oil and gas production, the fluid pressure in the development of oil and gas from low-permeable reservoirs decreases gradually with the production time. As a result, the rock skeleton, which bears the net overburden pressure of reservoirs, will be compressed and deformed. It can cause elastoplastic deformation of the rocks, and reduce the permeability of reservoir. Academically, it is defined as "stresssensitive reservoir". ${ }^{[18-23]}$ However, as far as we know, in previous researches of the moving boundary problems of fluid flow in low-permeable reservoir, the effect of deformation of low-permeable rock has not been considered and evaluated yet.
Here, based on these aforementioned concerns, in consideration of the two prominent physical phenomena in the development of low-permeable reservoirs i.e., the existence of TPG and the stress-sensitive effect, a new coupled moving boundary model of radial flow in low-permeable reservoir is developed; a concept of permeability modulus ${ }^{[18,19,21,22]}$ is introduced to reflect the effect of formation deformation on the permeability change. Besides, both the wellbore storage and skin effect are incorporated into the inner boundary conditions.

Abundant researches have been conducted on the analytical and numerical solutions for such moving boundary problems of fluid flow in porous medium with TPG. And current major solution methods include the integral approximate analytical method, ${ }^{[24]}$ the Green function method, ${ }^{[25]}$ the exact analytical solution by similarity transformation method, ${ }^{[17,26]}$ coordinate transformation based numerical method, ${ }^{[10]}$ and direct finite difference numerical method. ${ }^{[27]}$ Owing to the strong nonlinearity and complexity of our presented coupled moving boundary model, a coordinate transformation based finite difference method ${ }^{[10]}$ is adopted to numerically investigate the effect of physical parameters on the model solutions to the formation pressure and moving boundary. The utility of the numerical method can be attributed to its simple approach to converting the moving boundary problem into a problem with fixed boundary conditions, and then solving it by a stable fully implicit finite difference method on fixed spatial grids.

\section{Mathematical modeling}

\subsection{Physical model}

The problem considered involves the radial flow in an infinite low-permeable stress-sensitive reservoir with TPG. The reservoir is homogeneous, isotropic, and isothermal. And the single-phase horizontal flow does not have any gravity effect. Both the wellbore storage and skin effect ${ }^{[19]}$ are considered. The Newtonian fluid and rocks are slightly compressible.

The schematic of the physical model is shown in Fig. 2. Once the oil is produced from the low-permeable reservoir with TPG, the formation pressure drop happens in the formation inside the moving boundary. Owing to the formation pressure drop, the stress -sensitive reservoir can be deformed, which reduces the formation permeability (See Fig. 2).

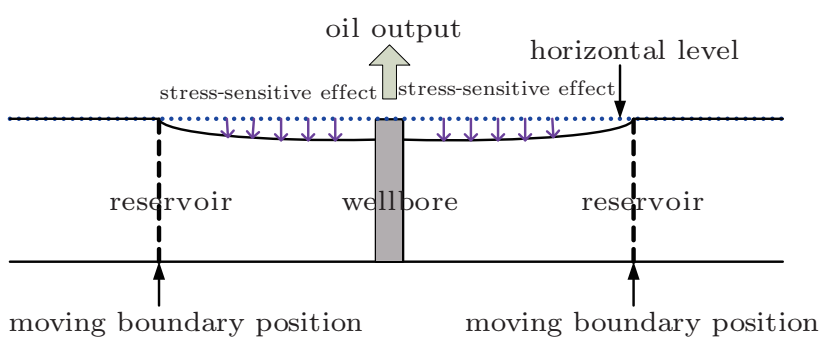

Fig. 2. (color online) Schematic diagram of physical model. 


\subsection{Mathematical model}

The fluid density is as follows: ${ }^{[24]}$

$$
\rho=\rho_{0} \exp \left(-C_{\mathrm{f}}\left(p_{0}-p\right)\right),
$$

where $\rho$ is the fluid density (in units $\mathrm{g} \cdot \mathrm{cm}^{-3}$ ); $\rho_{0}$ is the initial fluid density (in units $\mathrm{g} \cdot \mathrm{cm}^{-3}$ ); $p_{0}$ is the initial pressure (in unit atm); $p$ is the pressure (in unit atm, $\left.1 \mathrm{~atm}=1.01325 \times 10^{5} \mathrm{~Pa}\right) ; C_{\mathrm{f}}$ is the compression coefficient of the fluid (in unit $\mathrm{atm}^{-1}$ ).

The porosity of the porous medium is as follows: ${ }^{[24]}$

$$
\phi=\phi_{0} \exp \left(-C_{\phi}\left(p_{0}-p\right)\right)
$$

where $\phi$ is the porosity of the porous medium (in the fraction form); $\phi_{0}$ is the initial porosity (in the fraction form); $C_{\phi}$ is the compression coefficient of the porosity (in unit atm ${ }^{-1}$ ).

The modified Darcy's law for the fluid flow in the porous medium with TPG is as follows: ${ }^{[1]}$

$$
v= \begin{cases}-\frac{k}{\mu} \cdot \frac{\partial p}{\partial r} \cdot\left(1-\frac{\lambda}{\left.\left|\frac{\partial p}{\partial r}\right|\right),}\right. & \left|\frac{\partial p}{\partial r}\right|>\lambda, \\ 0, & 0 \leq\left|\frac{\partial p}{\partial r}\right| \leq \lambda,\end{cases}
$$

where $k$ is the permeability of the porous medium (in unit D); $\mu$ is the fluid viscosity (in unit $\mathrm{cP}$ ); $r$ is the radial distance (in unit $\mathrm{cm}$ ); $v$ is the seepage velocity (in units $\mathrm{cm}^{3} \cdot\left(\mathrm{s} \cdot \mathrm{cm}^{2}\right)^{-1}$ ); $\lambda$ is the TPG (in unis atm $\cdot \mathrm{cm}^{-1}$ ).

The permeability modulus $\gamma$ (in unit $\mathrm{atm}^{-1}$ ) is similar to the compressibility coefficient, and can be defined by the following equation: ${ }^{[18,19,21,22]}$

$$
\gamma=\frac{1}{k} \cdot \frac{\mathrm{d} k}{\mathrm{~d} p}
$$

Coefficient $\gamma$ plays an important role in the stresssensitive effect on the rock permeability. It is a measurement of the dependence of the permeability on formation pressure drop. For practical engineering applications, it can be assumed to be constant. ${ }^{[18,19,21,22]}$ Then the permeability of deformed rock in low-permeable reservoirs can be expressed from Eq. (4) as follows:

$$
k=k_{0} \mathrm{e}^{-\gamma\left(p_{0}-p\right)},
$$

where $k_{0}$ is the permeability at the initial reservoir pressure.

The continuous equation for the radial flow in the porous medium is as follows: ${ }^{[24]}$

$$
-\frac{1}{r} \frac{\partial}{\partial r}(r \rho v)=\frac{\partial(\rho \phi)}{\partial t}, \quad r_{\mathrm{w}} \leq r \leq s(t),
$$

where $t$ is the time (in unit s); $s$ is the moving boundary (in unit $\mathrm{cm}$ ); $r_{\mathrm{w}}$ is the wellbore radius (in unit $\mathrm{cm}$ ). Besides, it should be noted that Eq. (6) is only valid for the radial space from the wellbore to the moving boundary.

Substituting Eqs. (1)-(3), and (5) into Eq. (6), the governing (mass balance) equation for the radial flow in the lowpermeable stress-sensitive reservoir, can be deduced as follows:

$$
\begin{aligned}
& \frac{1}{r} \cdot \frac{\partial}{\partial r}\left[r \cdot \mathrm{e}^{-\gamma \cdot\left(p_{0}-p\right)} \cdot\left(\frac{\partial p}{\partial r}-\lambda\right)\right]=\frac{\mu \phi_{0} C_{\mathrm{t}}}{k_{0}} \cdot \frac{\partial p(r, t)}{\partial t} \\
& r_{\mathrm{w}} \leq r \leq s(t)
\end{aligned}
$$

where $C_{\mathrm{t}}$ is the total compression coefficient (in unit atm ${ }^{-1}$ ); and $C_{\mathrm{f}} \ll \gamma$.

The initial conditions are as follows:

$$
\begin{aligned}
& s(0)=0, \\
& \left.p\right|_{t=0}=p_{0} .
\end{aligned}
$$

The inner boundary conditions in consideration of wellbore storage and skin effect with constant flow rate are

$$
\begin{aligned}
& \left.\frac{2 \pi k_{0} h r}{\mu}\left(\frac{\partial p}{\partial r}-\lambda\right)\right|_{r=r_{\mathrm{w}}}=q \cdot B+C \cdot \frac{\mathrm{d} p_{\mathrm{wf}}}{\mathrm{d} t}, \\
& p_{\mathrm{wf}}=\left.\left[p-S \cdot \mathrm{e}^{-\gamma \cdot\left(p_{0}-p\right)} \cdot r \cdot\left(\frac{\partial p}{\partial r}-\lambda\right)\right]\right|_{r=r_{\mathrm{w}}},
\end{aligned}
$$

where $h$ is the reservoir thickness (in unit $\mathrm{cm}$ ); $C$ is the coefficient of wellbore storage (in units $\mathrm{cm}^{3} \cdot \mathrm{atm}^{-1}$ ); $q$ is the constant flow rate (in units $\mathrm{cm}^{3} \cdot \mathrm{s}^{-1}$ ); $B$ is the volume factor, dimensionless; $p_{\mathrm{wf}}$ is the wellbore pressure (in unit atm); $S$ is the skin factor.

According to the definition of TPG, the moving boundary conditions are the same as the ones in Ref. [10] as follows:

$$
\begin{aligned}
& \left.p\right|_{r=s(t)}=p_{0}, \\
& \left.\frac{\partial p}{\partial r}\right|_{r=s(t)}=\lambda .
\end{aligned}
$$

Equations (7)-(13) together form a coupled moving boundary model of radial flow in low-permeable stresssensitive reservoir with TPG for the case of a constant flow rate at the inner boundary in consideration of both the wellbore storage and skin effect.

The dimensionless variables are defined as follows:

$$
\begin{aligned}
& r_{\mathrm{D}}=\frac{r}{r_{\mathrm{w}}}, \\
& t_{\mathrm{D}}=\frac{k_{0} t}{\mu \phi_{0} C_{\mathrm{t}} r_{\mathrm{w}}^{2}}, \\
& \delta=\frac{s}{r_{\mathrm{w}}}, \\
& P_{\mathrm{D}}=\frac{2 \pi k_{0} h\left(p_{0}-p\right)}{q \mu B}, \\
& \lambda_{\mathrm{D}}=\frac{2 \pi k_{0} h r_{\mathrm{w}} \lambda}{q \mu B}, \\
& \gamma_{\mathrm{D}}=\frac{q \mu B \gamma}{2 \pi k_{0} h}, \\
& C_{\mathrm{D}}=\frac{C}{2 \pi \phi_{0} C_{\mathrm{t}} h r_{\mathrm{w}}^{2}},
\end{aligned}
$$




$$
P_{\mathrm{wD}}=\frac{2 \pi k_{0} h\left(p_{0}-p_{\mathrm{wf}}\right)}{q \mu B},
$$

where $r_{\mathrm{D}}$ is the dimensionless radial distance; $t_{\mathrm{D}}$ is the dimensionless time; $P_{\mathrm{D}}$ is the dimensionless pressure; $P_{\mathrm{wD}}$ is the dimensionless wellbore pressure; $\alpha_{\mathrm{D}}$ is the dimensionless compressibility; $\lambda_{D}$ is the dimensionless TPG; $\gamma_{\mathrm{D}}$ is the dimensionless permeability modulus; $\delta$ is the dimensionless moving boundary; $C_{\mathrm{D}}$ is the dimensionless coefficient of wellbore storage.

And then the dimensionless form of the coupled moving boundary model can be transformed equivalently. The governing equation is as follows:

$$
\begin{aligned}
& \frac{1}{r_{\mathrm{D}}} \cdot \frac{\partial}{\partial r_{\mathrm{D}}}\left[\mathrm{e}^{-\gamma_{\mathrm{D}} \cdot P_{\mathrm{D}}} \cdot r_{\mathrm{D}} \cdot\left(\frac{\partial P_{\mathrm{D}}}{\partial r_{\mathrm{D}}}+\lambda_{\mathrm{D}}\right)\right]=\frac{\partial P_{\mathrm{D}}\left(r_{\mathrm{D}}, t_{\mathrm{D}}\right)}{\partial t_{\mathrm{D}}}, \\
& 1 \leq r_{\mathrm{D}} \leq \delta\left(t_{\mathrm{D}}\right) .
\end{aligned}
$$

The initial conditions are as follows:

$$
\begin{aligned}
& \left.P_{\mathrm{D}}\right|_{t_{\mathrm{D}}=0}=0, \\
& \delta(0)=0 .
\end{aligned}
$$

The inner boundary conditions are as follows:

$$
\begin{aligned}
& C_{\mathrm{D}} \frac{\partial P_{\mathrm{wD}}}{\partial t_{\mathrm{D}}}-\left.\mathrm{e}^{-\gamma_{\mathrm{D}} \cdot P_{\mathrm{D}}} \cdot\left(\frac{\partial P_{\mathrm{D}}}{\partial r_{\mathrm{D}}}+\lambda_{\mathrm{D}}\right)\right|_{r_{\mathrm{D}}=1}=1, \\
& P_{\mathrm{wD}}=\left.\left[P_{\mathrm{D}}-S \cdot \mathrm{e}^{-\gamma_{\mathrm{D}} \cdot P_{\mathrm{D}}} \cdot\left(\frac{\partial P_{\mathrm{D}}}{\partial r_{\mathrm{D}}}+\lambda_{\mathrm{D}}\right)\right]\right|_{r_{\mathrm{D}}=1} .
\end{aligned}
$$

The moving boundary conditions are

$$
\begin{aligned}
& \left.\frac{\partial P_{\mathrm{D}}}{\partial r_{\mathrm{D}}}\right|_{r_{\mathrm{D}}=\delta\left(t_{\mathrm{D}}\right)}=-\lambda_{\mathrm{D}}, \\
& \left.P_{\mathrm{D}}\right|_{r_{\mathrm{D}}=\delta\left(t_{\mathrm{D}}\right)}=0 .
\end{aligned}
$$

\section{Velocity of moving boundary}

From Eq. (28), we have

$$
P_{\mathrm{D}}\left(\boldsymbol{\delta}\left(t_{\mathrm{D}}\right), t_{\mathrm{D}}\right)=0 .
$$

Differentiating two sides of Eq. (29) with respect to $t_{\mathrm{D}}$, we have

$$
\left.\frac{\partial P_{\mathrm{D}}}{\partial t_{\mathrm{D}}}\right|_{r_{\mathrm{D}}=\delta\left(t_{\mathrm{D}}\right)}+\left.\frac{\partial P_{\mathrm{D}}}{\partial r_{\mathrm{D}}}\right|_{r_{\mathrm{D}}=\delta\left(t_{\mathrm{D}}\right)} \cdot \frac{\partial \delta}{\partial t_{\mathrm{D}}}=0
$$

Substituting Eq. (27) into Eq. (30) yields

$$
\left.\frac{\partial P_{\mathrm{D}}}{\partial t_{\mathrm{D}}}\right|_{r_{\mathrm{D}}=\delta\left(t_{\mathrm{D}}\right)}=\lambda_{\mathrm{D}} \cdot \frac{\partial \delta}{\partial t_{\mathrm{D}}} .
$$

By using Eqs. (27) and (28) and letting $x_{\mathrm{D}}=\delta\left(t_{\mathrm{D}}\right)$ on both sides of Eq. (22), we have

$$
\left.\frac{\partial^{2} P_{\mathrm{D}}}{\partial r_{\mathrm{D}}^{2}}\right|_{r_{\mathrm{D}}=\delta\left(t_{\mathrm{D}}\right)}=\left.\frac{\partial P_{\mathrm{D}}}{\partial t_{\mathrm{D}}}\right|_{r_{\mathrm{D}}=\delta\left(t_{\mathrm{D}}\right)} .
$$

By Eqs. (31) and (32), the velocity of the moving boundary can be written as follows:

$$
\frac{\partial \delta}{\partial t_{\mathrm{D}}}=\left.\frac{1}{\lambda_{\mathrm{D}}} \frac{\partial^{2} P_{\mathrm{D}}}{\partial r_{\mathrm{D}}^{2}}\right|_{r_{\mathrm{D}}=\delta\left(t_{\mathrm{D}}\right)} .
$$

From Eq. (33), it can be concluded that the velocity of moving boundary is proportional to the second derivative of the formation pressure function with respect to the radial distance on the moving boundary, but inversely proportional to TPG. It is different from the result of classical heat-conduction Stefan problem. ${ }^{[15,17]}$

\section{Numerical method}

In order to overcome the difficulty in discretizing space for the transient flow region with moving boundary, a spatial coordinate transformation method is used. It is the same as the one for the radial seepage flow problem with moving boundary in Ref. [10] as follows:

$$
y\left(r_{\mathrm{D}}, t_{\mathrm{D}}\right)=\frac{r_{\mathrm{D}}-1}{\delta\left(t_{\mathrm{D}}\right)-1}, \quad 1 \leq r_{\mathrm{D}} \leq \boldsymbol{\delta}\left(t_{\mathrm{D}}\right) .
$$

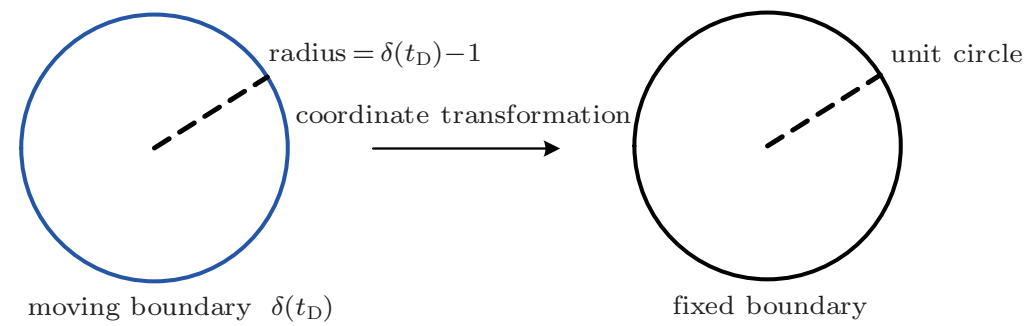

Fig. 3. (color online) Schematic diagram of coordinate transformation.

Through Eq. (34), the dynamic flow region for the moving boundary problem $\left[0, \delta\left(t_{\mathrm{D}}\right)\right]$ can be transformed into a fixed region $[0,1]$ as shown in Fig. 3 . Then the dimensionless pressure $P_{\mathrm{D}}\left(r_{\mathrm{D}}, t_{\mathrm{D}}\right)$ can be transformed into a new variable $\eta(y$,
$\left.t_{\mathrm{D}}\right)$. The same transformation of differential variables as the one in Ref. [10] can be obtained as

$$
\frac{\partial P_{\mathrm{D}}}{\partial r_{\mathrm{D}}}=\frac{\partial \eta}{\partial y} \cdot \frac{1}{(\boldsymbol{\delta}-1)},
$$




$$
\begin{aligned}
& \frac{\partial^{2} P_{\mathrm{D}}}{\partial r_{\mathrm{D}}^{2}}=\frac{\partial^{2} \eta}{\partial y^{2}} \cdot \frac{1}{(\delta-1)^{2}}, \\
& \frac{\partial P_{\mathrm{D}}}{\partial t_{\mathrm{D}}}=-\frac{\partial \eta}{\partial y} \cdot \frac{y}{(\delta-1)} \cdot \frac{\partial \delta}{\partial t_{\mathrm{D}}}+\frac{\partial \eta}{\partial t_{\mathrm{D}}} .
\end{aligned}
$$

Expanding the term on the left-hand side of the governing equation i.e., Eq. (22), the resulting equation can be obtained as follows:

$$
\begin{aligned}
& \frac{1}{r_{\mathrm{D}}} \cdot \frac{\partial P_{\mathrm{D}}}{\partial r_{\mathrm{D}}}+\frac{\lambda_{\mathrm{D}}}{r_{\mathrm{D}}}+\frac{\partial^{2} P_{\mathrm{D}}}{\partial r_{\mathrm{D}}^{2}} \\
& -\gamma_{\mathrm{D}} \cdot\left(\frac{\partial P_{\mathrm{D}}}{\partial r_{\mathrm{D}}}\right)^{2}-\lambda_{\mathrm{D}} \cdot \gamma_{\mathrm{D}} \cdot \frac{\partial P_{\mathrm{D}}}{\partial r_{\mathrm{D}}}=\frac{\partial P_{\mathrm{D}}}{\partial t_{\mathrm{D}}} \cdot \mathrm{e}^{\gamma_{\mathrm{D}} \cdot \eta} .
\end{aligned}
$$

Substituting Eqs. (35)-(37) into Eq. (38) yields

$$
\begin{aligned}
& \frac{1}{y_{\mathrm{D}} \cdot(\delta-1)+1} \cdot \frac{\partial \eta}{\partial y_{\mathrm{D}}} \cdot \frac{1}{(\delta-1)} \\
& +\frac{\lambda_{\mathrm{D}}}{y_{\mathrm{D}} \cdot(\delta-1)+1}+\frac{\partial^{2} \eta}{\partial y_{\mathrm{D}}^{2}} \cdot \frac{1}{(\delta-1)^{2}} \\
& -\gamma_{\mathrm{D}} \cdot\left(\frac{\partial \eta}{\partial y_{\mathrm{D}}} \cdot \frac{1}{(\delta-1)}\right)^{2}-\lambda_{\mathrm{D}} \cdot \gamma_{\mathrm{D}} \cdot \frac{\partial \eta}{\partial y_{\mathrm{D}}} \cdot \frac{1}{(\delta-1)} \\
= & \left(-\frac{\partial \eta}{\partial y_{\mathrm{D}}} \cdot \frac{y_{\mathrm{D}}}{(\delta-1)} \cdot \frac{\partial \delta}{\partial t_{\mathrm{D}}}+\frac{\partial \eta}{\partial t_{\mathrm{D}}}\right) \cdot \mathrm{e}^{\gamma_{\mathrm{D}} \cdot \eta} .
\end{aligned}
$$

Substituting Eqs. (35)-(37) into Eq. (23), Eqs. (25)-(28), and Eq. (33), respectively, we have

$$
\begin{aligned}
& \left.\eta\right|_{t_{\mathrm{D}}=0}=0 \\
& C_{\mathrm{D}} \frac{\partial P_{\mathrm{wD}}}{\partial t_{\mathrm{D}}}-\left.\mathrm{e}^{-\gamma_{\mathrm{D}} \cdot \eta} \cdot\left(\frac{\partial \eta}{\partial y_{\mathrm{D}}} \cdot \frac{1}{(\delta-1)}+\lambda_{\mathrm{D}}\right)\right|_{y_{\mathrm{D}}=0}=1, \\
& P_{\mathrm{wD}}=\left.\left[\eta-S \cdot \mathrm{e}^{-\gamma_{\mathrm{D}} \cdot \eta} \cdot\left(\frac{\partial \eta}{\partial y_{\mathrm{D}}} \cdot \frac{1}{(\delta-1)}+\lambda_{\mathrm{D}}\right)\right]\right|_{y_{\mathrm{D}}=0}, \\
& \left.\frac{\partial \eta}{\partial y}\right|_{y=1}=-\lambda_{\mathrm{D}}(\delta-1) \\
& \left.\eta\right|_{y=1}=0 \\
& \frac{\partial \delta}{\partial t_{\mathrm{D}}}=\left.\frac{1}{\lambda_{\mathrm{D}}} \frac{\partial^{2} \eta}{\partial y^{2}}\right|_{y=1} \cdot \frac{1}{(\delta-1)^{2}}
\end{aligned}
$$

From Eq. (43), we have

$$
\delta-1=-\left.\frac{1}{\lambda_{\mathrm{D}}} \cdot \frac{\partial \eta}{\partial y_{\mathrm{D}}}\right|_{y_{\mathrm{D}}=1} .
$$

Substituting Eqs. (45) and (46) into Eq. (39) to cancel the variables $\partial \delta / \partial t_{\mathrm{D}}$ and $\delta$ yields

$$
\begin{aligned}
& \frac{\partial \eta}{\partial y_{\mathrm{D}}} \cdot \frac{1}{\lambda_{\mathrm{D}}^{2}} \cdot\left(\left.\frac{\partial \eta}{\partial y_{\mathrm{D}}}\right|_{y_{\mathrm{D}}=1}\right)^{2}-\frac{1}{\lambda_{\mathrm{D}}^{2}} \cdot\left(\left.\frac{\partial \eta}{\partial y_{\mathrm{D}}}\right|_{y_{\mathrm{D}}=1}\right)^{3} \\
& +\frac{\partial^{2} \eta}{\partial y_{\mathrm{D}}^{2}} \cdot \frac{y_{\mathrm{D}}}{\lambda_{\mathrm{D}}^{2}} \cdot\left(\left.\frac{\partial \eta}{\partial y_{\mathrm{D}}}\right|_{y_{\mathrm{D}}=1}\right)^{2}-\left.\frac{1}{\lambda_{\mathrm{D}}} \cdot \frac{\partial^{2} \eta}{\partial y_{\mathrm{D}}^{2}} \cdot \frac{\partial \eta}{\partial y_{\mathrm{D}}}\right|_{y_{\mathrm{D}}=1} \\
& -\gamma_{\mathrm{D}} \cdot\left(\frac{\partial \eta}{\partial y_{\mathrm{D}}}\right)^{2} \cdot \frac{y_{\mathrm{D}}}{\lambda_{\mathrm{D}}^{2}} \cdot\left(\left.\frac{\partial \eta}{\partial y_{\mathrm{D}}}\right|_{y_{\mathrm{D}}=1}\right)^{2}
\end{aligned}
$$

$$
\begin{aligned}
& +\left.\frac{\gamma_{\mathrm{D}}}{\lambda_{\mathrm{D}}} \cdot\left(\frac{\partial \eta}{\partial y_{\mathrm{D}}}\right)^{2} \cdot \frac{\partial \eta}{\partial y_{\mathrm{D}}}\right|_{y_{\mathrm{D}}=1} \\
& +\gamma_{\mathrm{D}} \cdot \frac{\partial \eta}{\partial y_{\mathrm{D}}} \cdot \frac{y_{\mathrm{D}}}{\lambda_{\mathrm{D}}^{2}} \cdot\left(\left.\frac{\partial \eta}{\partial y_{\mathrm{D}}}\right|_{y_{\mathrm{D}}=1}\right)^{3} \\
& -\frac{\gamma_{\mathrm{D}}}{\lambda_{\mathrm{D}}} \cdot \frac{\partial \eta}{\partial y_{\mathrm{D}}} \cdot\left(\left.\frac{\partial \eta}{\partial y_{\mathrm{D}}}\right|_{y_{\mathrm{D}}=1}\right)^{2} \\
= & \left.\left.\frac{\partial \eta}{\partial y_{\mathrm{D}}} \cdot \frac{\partial^{2} \eta}{\partial y_{\mathrm{D}}^{2}}\right|_{y_{\mathrm{D}}=1} \cdot \mathrm{e}^{\gamma_{\mathrm{D}} \cdot \eta} \cdot \frac{y_{\mathrm{D}}^{2}}{\lambda_{\mathrm{D}}^{2}} \cdot \frac{\partial \eta}{\partial y_{\mathrm{D}}}\right|_{y_{\mathrm{D}}=1} \\
& -\left.\frac{\partial \eta}{\partial y_{\mathrm{D}}} \cdot \frac{y_{\mathrm{D}}}{\lambda_{\mathrm{D}}} \cdot \frac{\partial^{2} \eta}{\partial y_{\mathrm{D}}^{2}}\right|_{y_{\mathrm{D}}=1} \cdot \mathrm{e}^{\gamma_{\mathrm{D}} \cdot \eta} \\
& +\frac{\partial \eta}{\partial t_{\mathrm{D}}} \cdot \mathrm{e}^{\gamma_{\mathrm{D}} \cdot \eta} \cdot \frac{y_{\mathrm{D}}}{\lambda_{\mathrm{D}}^{4}} \cdot\left(\left.\frac{\partial \eta}{\partial y_{\mathrm{D}}}\right|_{y_{\mathrm{D}}=1}\right)^{4} \\
& -\frac{\partial \eta}{\partial t_{\mathrm{D}}} \cdot \mathrm{e}^{\gamma_{\mathrm{D}} \cdot \eta} \cdot \frac{1}{\lambda_{\mathrm{D}}^{3}} \cdot\left(\left.\frac{\partial \eta}{\partial y_{\mathrm{D}}}\right|_{y_{\mathrm{D}}=1}\right)^{3} .
\end{aligned}
$$

Substituting Eq. (46) into Eqs. (41) and (42) to cancel the variables $\delta$ yields

$$
\begin{aligned}
& \left.C_{\mathrm{D}} \cdot \frac{\partial P_{\mathrm{wD}}}{\partial t_{\mathrm{D}}} \cdot \frac{\partial \eta}{\partial y_{\mathrm{D}}}\right|_{y_{\mathrm{D}}=1}+\left.\lambda_{\mathrm{D}} \cdot\left(\mathrm{e}^{-\gamma_{\mathrm{D}} \cdot \eta} \cdot \frac{\partial \eta}{\partial y_{\mathrm{D}}}\right)\right|_{y_{\mathrm{D}}=0} \\
& -\left.\left.\mathrm{e}^{-\gamma_{\mathrm{D}} \cdot \eta}\right|_{y_{\mathrm{D}}=0} \cdot \lambda_{\mathrm{D}} \cdot \frac{\partial \eta}{\partial y_{\mathrm{D}}}\right|_{y_{\mathrm{D}}=1}=\left.\frac{\partial \eta}{\partial y_{\mathrm{D}}}\right|_{y_{\mathrm{D}}=1}, \\
& \left.P_{\mathrm{wD}} \cdot \frac{\partial \eta}{\partial y_{\mathrm{D}}}\right|_{y_{\mathrm{D}}=1} \\
& =\left.\left.\eta\right|_{y_{\mathrm{D}}=0} \cdot \frac{\partial \eta}{\partial y_{\mathrm{D}}}\right|_{y_{\mathrm{D}}=1}+\left.\left.S \cdot \lambda_{\mathrm{D}} \cdot \mathrm{e}^{-\gamma_{\mathrm{D}} \cdot \eta}\right|_{y_{\mathrm{D}}=0} \cdot \frac{\partial \eta}{\partial y_{\mathrm{D}}}\right|_{y_{\mathrm{D}}=0} \\
& -\left.\left.S \cdot \lambda_{\mathrm{D}} \cdot \mathrm{e}^{-\gamma_{\mathrm{D}} \cdot \eta}\right|_{y_{\mathrm{D}}=0} \cdot \frac{\partial \eta}{\partial y_{\mathrm{D}}}\right|_{y_{\mathrm{D}}=1} \cdot
\end{aligned}
$$

Equations (47)-(49), Eq. (40) and Eq. (44) together form a transformed mathematical model with fixed boundary conditions. The model shows strong nonlinearity, indirectly indicating the strong nonlinearity of the original, untransformed moving boundary problem. Here, a stable, fully implicit finite difference method ${ }^{[10]}$ is used to obtain its numerical solutions of the nonlinear model. The difference equation for the model can be written as follows:

$$
\begin{aligned}
& \frac{\eta_{i+1}^{j+1}-\eta_{i}^{j+1}}{\Delta y} \cdot \frac{1}{\lambda_{\mathrm{D}}^{2}} \cdot\left(-\frac{\eta_{N-1}^{j+1}}{\Delta y}\right)^{2}-\frac{1}{\lambda_{\mathrm{D}}^{2}} \cdot\left(-\frac{\eta_{N-1}^{j+1}}{\Delta y}\right)^{3} \\
& +\frac{\eta_{i-1}^{j+1}-2 \eta_{i}^{j+1}+\eta_{i+1}^{j+1}}{(\Delta y)^{2}} \cdot \frac{i \Delta y_{\mathrm{D}}}{\lambda_{\mathrm{D}}^{2}} \cdot\left(-\frac{\eta_{N-1}^{j+1}}{\Delta y}\right)^{2} \\
& -\frac{1}{\lambda_{\mathrm{D}}} \cdot \frac{\eta_{i-1}^{j+1}-2 \eta_{i}^{j+1}+\eta_{i+1}^{j+1}}{(\Delta y)^{2}} \cdot\left(-\frac{\eta_{N-1}^{j+1}}{\Delta y}\right) \\
& -\gamma_{\mathrm{D}} \cdot\left(\frac{\eta_{i+1}^{j+1}-\eta_{i}^{j+1}}{\Delta y}\right)^{2} \frac{\mathrm{i} \Delta y_{\mathrm{D}}}{\lambda_{\mathrm{D}}^{2}} \cdot\left(-\frac{\eta_{N-1}^{j+1}}{\Delta y}\right)^{2} \\
& +\frac{\gamma_{\mathrm{D}}}{\lambda_{\mathrm{D}}} \cdot\left(\frac{\eta_{i+1}^{j+1}-\eta_{i}^{j+1}}{\Delta y}\right)^{2} \cdot\left(-\frac{\eta_{N-1}^{j+1}}{\Delta y}\right)
\end{aligned}
$$




$$
\begin{aligned}
& +\gamma_{\mathrm{D}} \cdot \frac{\eta_{i+1}^{j+1}-\eta_{i}^{j+1}}{\Delta y} \cdot \frac{\mathrm{i} \Delta y_{\mathrm{D}}}{\lambda_{\mathrm{D}}^{2}} \cdot\left(-\frac{\eta_{N-1}^{j+1}}{\Delta y}\right)^{3} \\
& -\frac{\gamma_{\mathrm{D}}}{\lambda_{\mathrm{D}}} \cdot \frac{\eta_{i+1}^{j+1}-\eta_{i}^{j+1}}{\Delta y} \cdot\left(-\frac{\eta_{N-1}^{j+1}}{\Delta y}\right)^{2} \\
& -\frac{\eta_{i+1}^{j+1}-\eta_{i}^{j+1}}{\Delta y} \cdot \frac{\eta_{N-2}^{j+1}-2 \eta_{N-1}^{j+1}}{(\Delta y)^{2}} \\
& \cdot \mathrm{e}^{\gamma_{\mathrm{D}} \cdot \eta_{i}^{j+1}} \cdot \frac{\left(\mathrm{i} \Delta y_{\mathrm{D}}\right)^{2}}{\lambda_{\mathrm{D}}^{2}} \cdot\left(-\frac{\eta_{N-1}^{j+1}}{\Delta y}\right) \\
& +\frac{\eta_{i+1}^{j+1}-\eta_{i}^{j+1}}{\Delta y} \cdot \frac{i \Delta y_{\mathrm{D}}}{\lambda_{\mathrm{D}}} \cdot \frac{\eta_{N-2}^{j+1}-2 \eta_{N-1}^{j+1}}{(\Delta y)^{2}} \cdot \mathrm{e}^{\gamma_{\mathrm{D}} \cdot \eta_{i}^{j+1}} \\
& -\frac{\eta_{i}^{j+1}-\eta_{i}^{j}}{\Delta t} \cdot \mathrm{e}^{\gamma_{\mathrm{D}} \cdot \eta_{i}^{j+1}} \cdot \frac{i \Delta y_{\mathrm{D}}}{\lambda_{\mathrm{D}}^{4}} \cdot\left(-\frac{\eta_{N-1}^{j+1}}{\Delta y}\right)^{4} \\
& +\frac{\eta_{i}^{j+1}-\eta_{i}^{j}}{\Delta t} \cdot \mathrm{e}^{\gamma_{\mathrm{D}} \cdot \eta_{i}^{j+1}} \cdot \frac{1}{\lambda_{\mathrm{D}}^{3}} \cdot\left(-\frac{\eta_{N-1}^{j+1}}{\Delta y}\right)^{3}=0, \\
& i=1,2, \ldots, N-1 \text {, } \\
& -C_{\mathrm{D}} \cdot \frac{P_{\mathrm{wD}}^{j+1}-P_{\mathrm{wD}}^{j}}{\Delta t} \cdot \frac{\eta_{N-1}^{j+1}}{\Delta y}+\lambda_{\mathrm{D}} \cdot \mathrm{e}^{-\gamma_{\mathrm{D}} \cdot \eta_{0}^{j+1}} \cdot \frac{\eta_{1}^{j+1}-\eta_{0}^{j+1}}{\Delta y} \\
& +\mathrm{e}^{-\gamma_{\mathrm{D}} \cdot \eta_{0}^{j+1}} \cdot \lambda_{\mathrm{D}} \cdot \frac{\eta_{N-1}^{j+1}}{\Delta y}+\frac{\eta_{N-1}^{j+1}}{\Delta y}=0, \\
& -P_{\mathrm{wD}}^{j+1} \cdot \frac{\eta_{N-1}^{j+1}}{\Delta y}+\eta_{0}^{j+1} \cdot \frac{\eta_{N-1}^{j+1}}{\Delta y} \\
& -S \cdot \lambda_{\mathrm{D}} \cdot \mathrm{e}^{-\gamma_{\mathrm{D}}} \cdot \eta_{0}^{j+1} \cdot \frac{\eta_{1}^{j+1}-\eta_{0}^{j+1}}{\Delta y} \\
& -S \cdot \lambda_{\mathrm{D}} \cdot \mathrm{e}^{-\gamma_{\mathrm{D}} \cdot \eta_{0}^{j+1}} \cdot \frac{\eta_{N-1}^{j+1}}{\Delta y}=0, \\
& \eta_{i}^{0}=0, \quad i=0,1,2, \ldots, N-1, \\
& \eta_{N}^{j+1}=0,
\end{aligned}
$$

where $N$ denotes the total number of spatial grid subintervals with the same length; $\Delta y$ is the length of a grid subinterval, which is equal to $1 / N ; i$ denotes the index of the spatial grid from the well; $j$ denotes the index of a time step; $\Delta t_{\mathrm{D}}$ denotes the time step size.

Equations (50)-(54) together form the closed difference equations at the $(j+1)$-th time step, and also contain $(N+1)$ unknown variables. The Newton-Raphson iterative method $^{[10]}$ is used to numerically solve these nonlinear difference equations. Eventually, numerical solutions for the transformed nonlinear partial differential equation system with respect to $\eta\left(y, t_{\mathrm{D}}\right)$ can be obtained.

The difference equation of Eq. (46) is

$$
\delta^{j+1}-1=\frac{1}{\lambda_{\mathrm{D}}} \cdot \frac{\eta_{N-1}^{j+1}}{\Delta y} .
$$

Substituting Eq. (55) into the difference equation of Eq. (34) yields

$$
r_{\mathrm{D} i}^{j+1}=i \cdot \frac{\eta_{N-1}^{j+1}}{\lambda_{\mathrm{D}}}+1
$$

By Eq. (56), numerical solutions of $\eta\left(y, t_{\mathrm{D}}\right)$ can be transformed into the ones of $P_{\mathrm{D}}\left(r_{\mathrm{D}}, t_{\mathrm{D}}\right)$ in the numerical solution process.

\section{Verification of numerical solutions}

$\mathrm{Li}$ and $\mathrm{Liu}^{[27]}$ have studied a moving boundary model of radial flow in porous medium with TPG without consideration of the stress-sensitive effect, nor wellbore storage nor skin effect. Its numerical solution is obtained by direct spatial discretization with fixed grids. In our presented coupled moving boundary model, if $\gamma_{\mathrm{D}}, C_{\mathrm{D}}$, and $S$ are all set to be zero, the coupled moving boundary model can be reduced to the same model as the one in Ref. [27]. From Eq. (55), the dimensionless moving boundary distance $\delta$ can be computed for each time step. Then the numerical solutions regarding the dimensionless transient distance of moving boundary by the two different numerical methods can be compared with others.

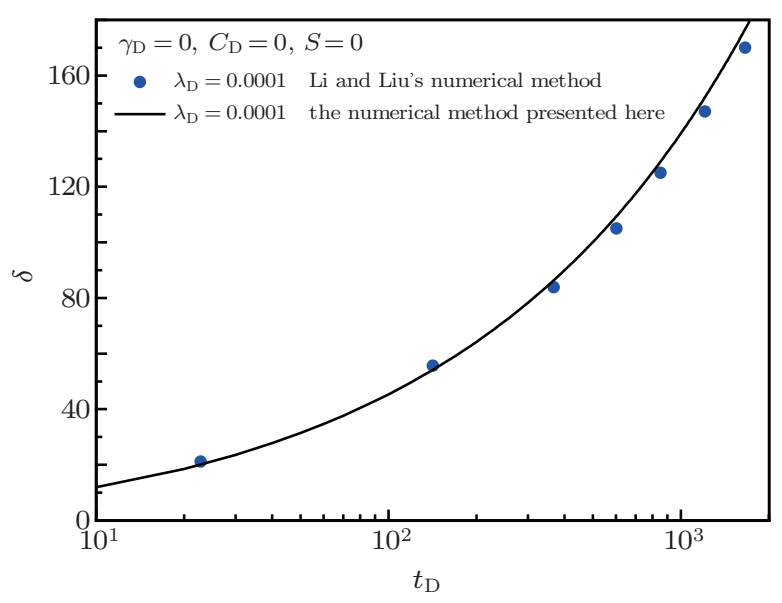

Fig. 4. (color online) Comparison of numerical results

Figure 4 shows the comparison between numerical results when the value of dimensionless TPG $\lambda_{\mathrm{D}}$ is equal to 0.0001 . From Fig. 4, it can be seen that the two numerical solutions are in a good agreement. Therefore, the validity of the coordinate transformation based finite difference numerical method of solving the coupled moving boundary model presented here can be verified.

\section{Results and discussion}

\subsection{Effect of dimensionless permeability modulus $\gamma_{D}$}

Figure 5 shows the effects of dimensionless permeability modulus on the log-log curves of dimensionless transient wellbore pressure and its derivative (with respect to dimensionless time) multiplied by the dimensionless time, under different values of dimensionless permeability modulus. These typical log-log curves are very useful for the well testing explanation in petroleum engineering. Figure 6 shows the effects 
of dimensionless permeability modulus on the dimensionless formation pressure distribution for different values of $\gamma_{D}$.

Figure 5 shows that the dimensionless permeability modulus, characterizing the reservoir deformation, has little effect on both the transient wellbore pressure and its derivative.

Figure 6 indicates that the larger the dimensionless radial distance, the bigger the effect of reservoir deformation on the formation pressure is; the larger the dimensionless permeability modulus, the nearer the dimensionless distance of moving boundary is. It can be explained that the reservoir deformation can reduce the formation permeability as the formation pressure drops, which slows down the propagation of moving boundary. Besides, it can be concluded from Fig. 6 that the larger the dimensionless permeability modulus, the bigger the sensitive effect of the parameter is.

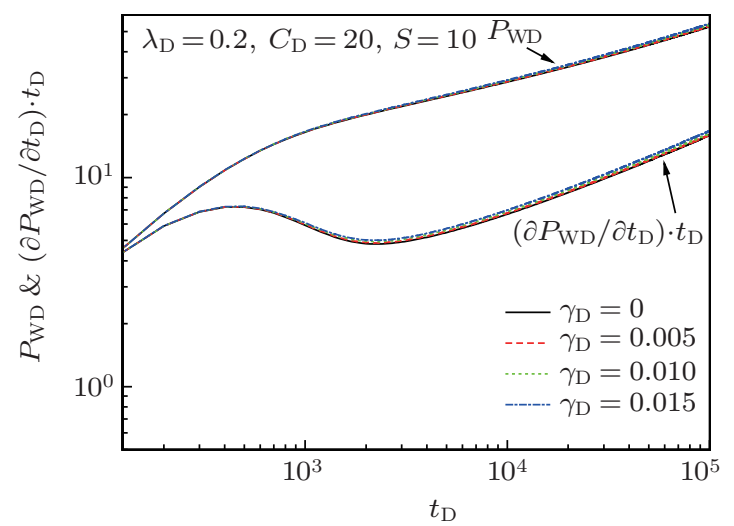

Fig. 5. (color online) Effects of dimensionless permeability modulus on transient wellbore pressure and its derivative for different values of $\gamma_{D}$.

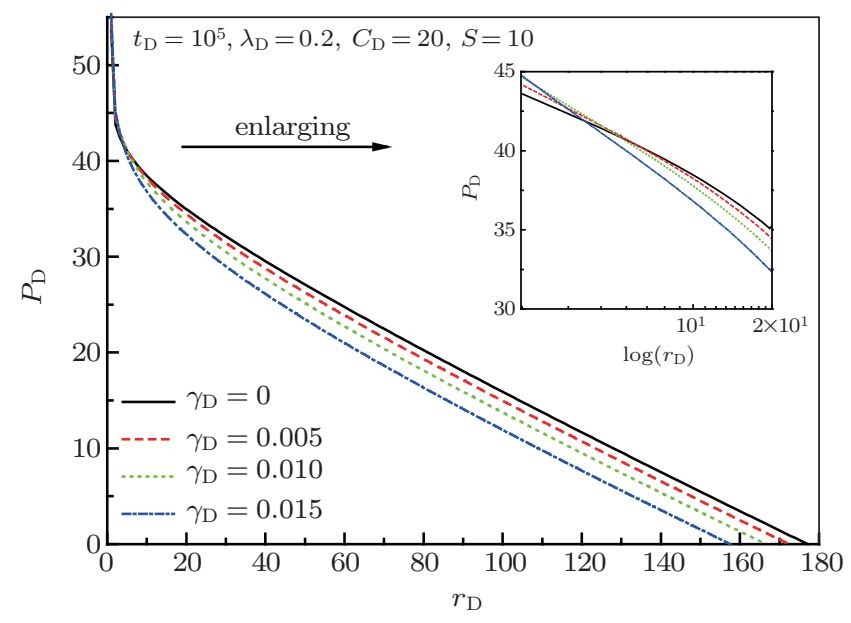

Fig. 6. (color online) Effects of dimensionless permeability modulus on formation pressure distribution for different values of $\gamma_{D}$

With respect to the dimensionless formation pressure, near the wellbore (see the enlarged semi-log plot in the inset of Fig. 6), with the increase of the dimensionless radial distance, the larger the dimensionless permeability modulus, the larger the dimensionless formation pressure and the absolute value of the dimensionless formation pressure gradient are; however, in the place further away from the wellbore, with the increase of the dimensionless radial distance, the larger the dimensionless permeability modulus, the smaller the dimensionless formation pressure is. Then it can be concluded that the more serious pressure drop caused by the formation deformation in low-permeable reservoirs mainly happens in the place near the wellbore; it can be explained that much larger formation pressure drop happens near the wellbore than far away from the wellbore in the formation, and can cause greater formation deformation and subsequent permeability reduction.

\subsection{Effect of dimensionless TPG $\lambda_{D}$}

Figures 7 and 8 show the effects of dimensionless TPG $\lambda_{\mathrm{D}}$ on dimensionless transient wellbore pressure and its derivative, and dimensionless formation pressure distribution respectively, under different values of dimensionless TPG. The estimation of value range of $\lambda_{D}$ has been performed from the experimental data of eight samples ${ }^{[1,10]}$ and the range is from 0 to 0.852 . Here four different values of $\lambda_{\mathrm{D}}$ are set to be 0.1 , $0.2,0.3$, and 0.4 , respectively.

From Figs. 7 and 8, it can be seen that the larger the dimensionless TPG, the larger the dimensionless transient wellbore pressure and its derivative are and the smaller the disturbed area between the wellbore and the position of moving boundary is. In comparison with the typical curves of Darcy's flow, these log-log curves, which correspond to nonzero value of TPG, all turn upward, and the line representing the period of radial flow is not horizontal any more. The formation pressure distribution curve corresponding to larger TPG exhibits a larger slope. Moreover, unlike for Darcy's flow problem, for this moving boundary problem, the formation pressure drops only inside the moving boundary, but outside the moving boundary the formation still keeps initial pressure: the pressure distribution curves show a property of compact support. $^{[10]}$

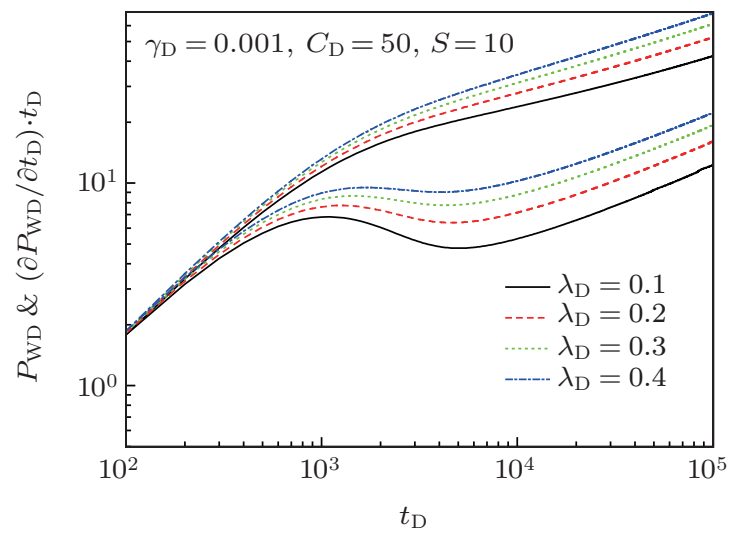

Fig. 7. (color online) Effects of dimensionless TPG on transient wellbore pressure and its derivative.

Besides, from Fig. 7, it can also be concluded that the smaller the dimensionless TPG, the bigger the sensitive effects on the transient wellbore pressure and the derivative are, 
which challenges the well testing problems in low-permeable reservoirs.

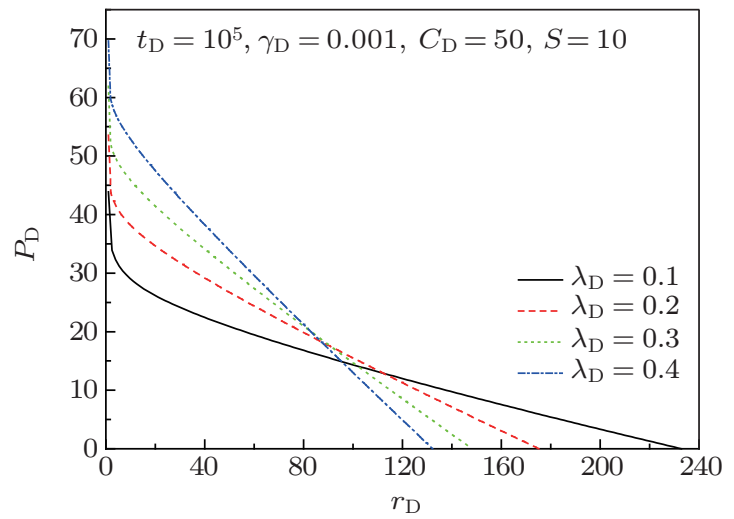

Fig. 8. (color online) Effect of dimensionless TPG on formation pressure distribution.

\subsection{Effect of dimensionless wellbore storage coefficient $C_{\mathrm{D}}$}

Figures 9 and 10 show the effects of dimensionless wellbore storage $C_{\mathrm{D}}$ on dimensionless transient wellbore pressure and derivative and dimensionless formation pressure distribution respectively, under different values of dimensionless wellbore storage coefficient $C_{\mathrm{D}}$.

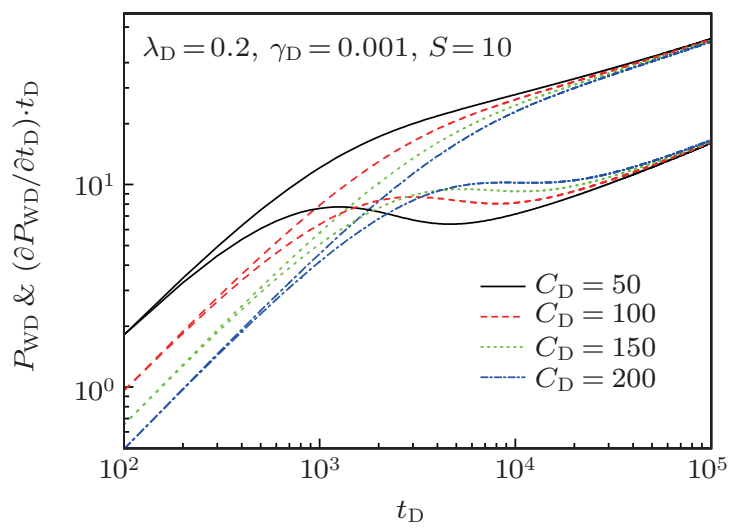

Fig. 9. (color online) Effects of dimensionless wellbore storage coefficient on transient wellbore pressure and its derivative for different values of $C_{\mathrm{D}}$.

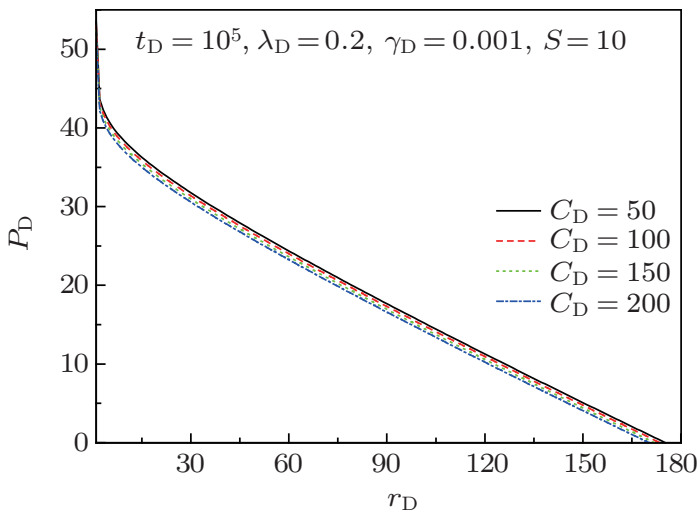

Fig. 10. (color online) Effects of dimensionless wellbore storage coefficient on formation pressure distribution for different values of $C_{\mathrm{D}}$.

From Fig. 9, it can be seen that the wellbore storage mainly affects the initial stage of transient wellbore pressure behavior, and with time increasing, its effect tends to diminish. The smaller the dimensionless wellbore storage coefficient, the less the time of wellbore storage is and the larger the sensitive effect is. From Fig. 10, it can also be concluded that the effect of dimensionless wellbore storage coefficient on dimensionless formation pressure distribution is not very obvious. The larger the dimensionless wellbore storage coefficient, the smaller the dimensionless transient wellbore pressure and the dimensionless formation pressure are.

\subsection{Effect of skin factor $S$}

Figures 11 and 12 show the effects of skin factor $S$ on dimensionless transient wellbore pressure and its derivative and dimensionless formation pressure distribution respectively, under different values of skin factor $S$. From Figs. 11 and 12 , it can be seen that the effect of skin factor on the dimensionless transient wellbore pressure is more obvious than on the dimensionless formation pressure distribution. Its effect on the derivative is more serious in the early production period. The larger the skin factor, the larger the dimensionless transient wellbore pressure and its derivative are, and with time increasing, its effect tends to diminish. In addition, the skin factor has little effect on the dimensionless formation pressure distribution.

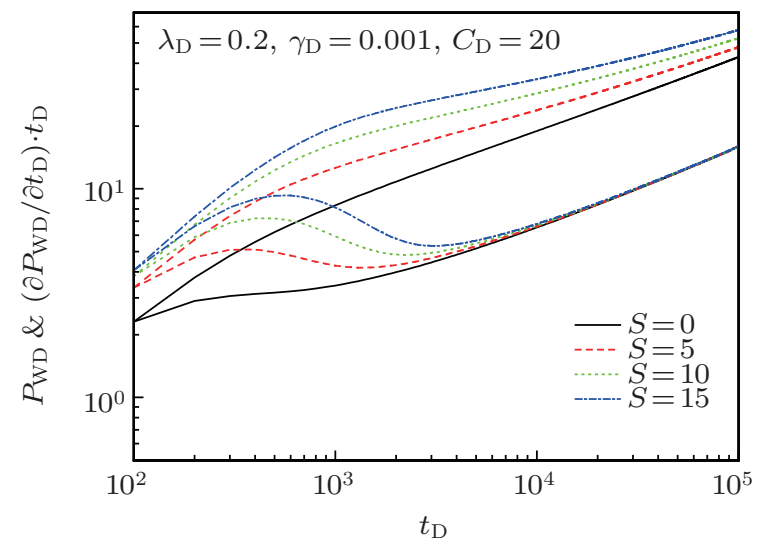

Fig. 11. (color online) Effects of skin factor on transient wellbore pressure and its derivative for different values of skin factor $S$.

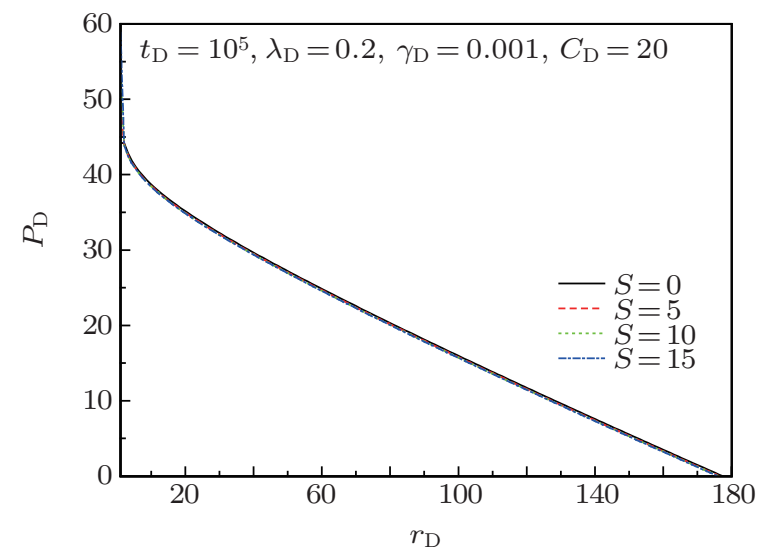

Fig. 12. (color online) Effects of skin factor on the formation pressure distribution for different values of skin factor $S$. 


\section{Conclusions}

A new coupled moving boundary model of radial flow in a stress-sensitive reservoir with TPG is built in consideration of the aforementioned two prominent physical phenomena in the development of low-permeable reservoir. A coordinate transformation based fully implicit finite difference method is adopted to obtain the numerical solutions of the nonlinear model. Its validity is also verified.

Some important conclusions from the numerical results analysis are obtained. The dimensionless permeability modulus, indicating the reservoir deformation, has little effect on transient wellbore pressure and its derivative. The more serious pressure drop caused by the formation deformation in low-permeable reservoirs mainly happens in the place near the wellbore. Unlike the scenario from the Darcy's flow problem, the formation pressure distribution curves corresponding to nonzero TPG show a property of compact support; the loglog typical curves all turn upward, and the line representing the period of radial flow is not horizontal. The effect of dimensionless wellbore storage coefficient on the dimensionless formation pressure distribution is not very obvious, and it mainly affects the initial stage of transient wellbore pressure behavior. The skin factor has little effect on the dimensionless formation pressure distribution.

\section{References}

[1] Prada A and Civan F 1999 J. Pet. Sci. Eng. 22237
[2] Song F Q, Jiang R J and Bian S L 2007 Chin. Phys. Lett. 241995

[3] Xiong W, Lei Q, Gao S S, Hu Z M and Xue H 2009 Petroleum Exploration and Development 36232

[4] Yue X A, Wei H G, Zhang L J, Zhao R B and Zhao Y P 2010 Transport Porous Med. 85333

[5] Yu R Z, Lei Q, Yang Z M and Bian Y N 2010 Chin. Phys. Lett. 27 024704

[6] Song F Q, Wang J D and Liu H L 2010 Chin. Phys. Lett. 27024704

[7] Yao Y and Ge J 2011 Pet. Sci. 855

[8] Cai J C and Yu B M 2011 Transport Porous Med. 89251

[9] Guo J J, Zhang S, Zhang L H, Qing H R and Liu Q G 2012 Journal of Hydrodyn. Ser. B 24561

[10] Yao J, Liu W C and Chen Z X 2013 Math. Probl. Eng. 2013384246

[11] Cai J C 2014 Chin. Phys. B 23044701

[12] Cai J C, Perfect E, Cheng C L and Hu X Y 2014 Langmuir 305142

[13] Tan X H, Li X P, Zhang L H, Liu J Y and Cai J C 2015 Int. J. Mod. Phys. C 261550045

[14] Zhu W Y, Song H Q, Huang X H, Liu X, He D B and Ran Q Q 2011 Energy \& Fuels 251111

[15] Zhu Y, Xie J Z, Yang W H and Hou L H 2008 Petroleum Exploration and Development 35225

[16] Yin D Y and Pu H 2008 J. Hydrodyn. Ser. B 20492

[17] Liu W C, Yao J and Wang Y Y 2012 Int. J. Heat Mass Transfer 556017

[18] Wang H, Wang G, Chen Z and Wong R C K 2010 J. Petrol. Sci. Eng. 75240

[19] Zhang L H, Guo J J and Liu Q G 2010 Pet. Sci. 7524

[20] Jasinge D, Ranjith P G and Choi S K 2011 Fuel 901292

[21] Zhang L H, Guo J J and Liu Q G 2011 J. Hydrodyn. Ser. B 23759

[22] Wu Y S and Pruess K 2000 Int. J. Rock Mech. Min. Sci. 3751

[23] Chen D, Pan Z and Ye Z 2015 Fuel 139383

[24] Wu Y S, Pruess K and Witherspoon P A 1992 SPE Reservoir Engineering 7369

[25] Lu J 2012 Special Topics \& Reviews in Porous Media-An International Journal 3307

[26] Liu W C, Yao J and Chen Z X 2014 Acta Mech. Sin. 3050

[27] Li F H and Liu C Q 1997 Well Testing 61 